\title{
Consumer Information Services in Intercultural Tourism: An Ethnographic Study of Chinese Outbound Backpackers
}

\author{
Brad McKenna \\ University of East Anglia \\ b.mckenna@uea.ac.uk
}

\author{
Wenjie Cai \\ University of Surrey \\ w.cai@surrey.ac.uk
}

\author{
Tuure Tuunanen \\ University of Jyväskylä \\ tuure@tuunanen.fi
}

\begin{abstract}
This paper reports the findings of an ethnographic study of Chinese outbound backpackers' use and adoption of consumer information services (CIS) in an intercultural tourism setting. We apply McKenna et al.'s research model of consumers' adoption of information services as the analytical lens for the interpretive qualitative study. The data gathering was conducted in four different countries. The findings of the study confirm linkages between four information service types and the use and adoption of CIS. The study also found that service types are more diversely linked than the earlier studies have predicted and therefore we propose a revised research model, which can be used for studying different CIS usage behaviour/patterns, but also to design of CIS for specific contexts.
\end{abstract}

\section{Introduction}

Backpacker sociality is a networking sociality, which is created through intersecting movements between physical and virtual space [2]. Interweaving with physical sociality, Chinese backpackers (CBs) highly rely on technologies and are active on the virtual world. This study, focuses on connectedness of $\mathrm{CBs}$ and their daily social networks through Information Communication Technology (ICT) enabled services [3], and more specifically consumer information services (CIS). CIS is defined by [4]: " $a$ s systems that enable consumer value co-creation through the development and implementation of information technology enabled processes that integrate system value propositions with customer value drivers to provide ICT-enabled services" (p.48).

This paper aims to explore the link between service types and technology usage to understand how users receive value from using CIS, focusing on a users' perspective of CBs' perceptions and adoptions of CIS. To achieve this aim, we investigate the CIS use by Chinese outbound backpackers in Europe, by exploring their perceptions, practice and preferences in an intercultural context.
The Confucian value of Harmony, has been regarded as the foundation of Chinese values and significantly impact the pattern of social interactions [5]. The influence mainly reflected in the determination of built up connections called 'guanxi', which is a fundamental factor in Chinese society [6]. Chinese believe the establishment of guanxi in the initial stage of group communication has vital impact on the harmonious interactions.

According to Reisinger and Turner [7], Chinese cultures are regarded as high power distance and collectivistic cultures whilst Western cultures are opposite. In terms of power distance, Chinese society tends to be more socially hierarchical, obedient and cooperative. Furthermore, with the influence of collectivism, Hsu [8] emphasizes Chinese are social and psychological dependent on others and have a strong group orientation; therefore, individualistic behaviour is regarded as expense to others. Reisinger and Turner [7] conclude that in Chinese culture, there are more rules of obedience, avoidance of conflict, and 'face' issues, it is essential to maintain harmonious relations, and Chinese tend to control and restrain emotional expressions. Chinese culture and values influence significantly on the formations of attitudes, perceptions and behaviours, leads the distinct characteristics of CBs. This makes $\mathrm{CBs}$ a very interesting research domain for our ethnographic study.

This paper gives us the opportunity to explore CIS in an intercultural tourism context and our research contributes to the CIS literature by extending the research model of McKenna et al. [1] which contains proposed linkages between service types and usage variables. This study will test those propositions with data from a study of CBs who tend to use a suite of technologies and applications. This differs from the McKenna et al model which was created through the design of one mobile application. Furthermore, the model proposes that when particular ICT service types are being used by consumers, that certain use and acceptance variables also apply to the use of that CIS. Consequently, our research question is: how do users receive value from using CIS, especially in terms of 
Self-Efficacy, Social Influences, and Facilitating Conditions?

The structure of the paper is following. We first review selected set of the CIS literature to provide the foundation for our ethnographic study. Then we present the research methodology, the analysis and results of our field study. Therefore, we discuss the findings and conclude.

\section{CIS Use by Chinese Backpackers}

CBs rely highly on CIS during travel [9]. Molz [10] argues the visibility in this social relation incorporated by online travel websites: On one hand, an online audience can see the world through travellers' gaze by formats of photography, video and texts; on the other hand, backpackers make themselves visible through various ways. The issue of surveillance has been addressed in discussions between mobilities and ICT $[10,11]$. Backpackers are watched by their audience through social media or online forums, which brings both positive and negative repercussion for mobile social relations [12].

Having a constant presence online can be seen as positive, which allows continual communication for backpackers, who are hypermobile and do not follow planned itineraries. However, this constant presence might also make backpackers feel oppressive. For some backpackers, with the influence of one-child policy, they are expected to keep updates in order to appease worried relatives and friends. These updates thus become supplied surveillance from their parents to know where they are and know whether they are safe or not, which to some extent limit their freedom of travelling. Backpackers can always be contacted by any means of social media or instant messenger, therefore, they can never hide or escape from this implied surveillance. Molz [10] suggests the expectation of visibility and availability by audience through online social networks may exacerbate rather than appease.

It therefore becomes necessary for us to understand how CIS are being used in this context. To better understand how consumers perceive and derive value from the ICT services they use, McKenna et al. [1] derived a model (cf. Figure 1) which combines the theory of organizational information services (TOIS) [13] with the unified theory of acceptance and use of technology (UTAUT) [14]. TOIS defines the information service types as follows:

- Computational service components support users in transforming available and formalized information into stimuli by following standardized and repeatable patterns of information processing.

- Adaptive service components interpret and transform available and emergent information into stimuli by adapting patterns of information processing to specific contexts.

- Networking service components aid users in producing information on phenomena in an environment by following standardized and repeatable patterns of information processing.

- Collaborative service components support users in producing information about phenomena in an environment through interpretation of the specific context $[1,13]$.

The UTAUT model defines the variables as follows:

- Self-efficacy: the judgment of one's ability to use a technology to accomplish a particular job or task.

- Social influence: the degree to which an individual perceives that important others believe that he or she should use the new system.

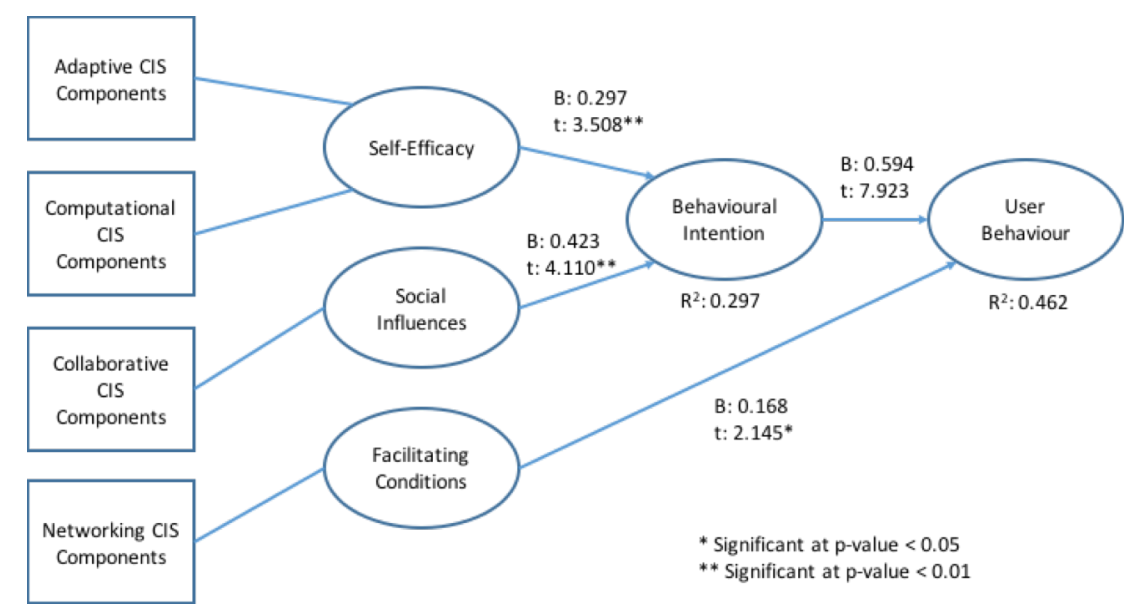

Figure 1: Research model by McKenna et al. [1] for consumers' adoption of information services. 
- Facilitating conditions: the degree to which an individual believes that an organizational and technical infrastructure exists to support use of the system [14].

The research model (Figure 1) was developed based on several propositions, and the actual mapping of services to the UTAUT variables has not been explored. However, McKenna et al.'s [1] results show that distinct service components can be potentially linked to specific UTAUT constructs. Their results also are showed that TOIS can be utilized within a CIS context and that these four service types (adaptive, collaborative, computational, and networking) should be considered when designing CIS.

More specifically, McKenna et al. [1] argue that for CIS, which the consumer needs to do something (computational and adaptive CIS components), the designers must ensure that these services are selfefficient, i.e., that consumers must be able to complete the tasks on their own. Collaborative CIS components are, in turn, impacted by the social influences of the technologies utilized in the service, and networking service components are impacted by the conditions that facilitate their use, as well as the previous experience of the consumer. McKenna et al. 1] designed an artefact (a prototype) through which the research model was tested. The more comprehensive model was reduced to what is presented in Figure 1. The research model was tested with a controlled experiment. The experiment participants used the artefact, and afterwards, answered an online questionnaire about their use experiences.

The results of the experiment are summarised in Figure 1. These showed a statistical significance between all tested constructs at 0.01 level except one, which was statistically significant at 0.05 level (facilitating conditions $\rightarrow$ use behaviour). The experiment and the results are depicted at more detail level in [1]. In this paper, we explore the propositions made by McKenna et al (the left side of the model).

\section{Research Methodology}

The philosophical stance of this study is interpretive which assumes that reality is accessed through social constructions such as language, consciousness and shared meanings. Interpretive methods of research in IS are "aimed at producing an understanding of the context of the information system, and the process whereby the information system influences and is influenced by the context" (p.4-5) [15]. Interpretive researchers do not aim at "falsifying" theories, but tend to use theories as a "sensitizing device" which allows the researcher to view the world in a particular way [16]. In this study, we have used the research model of McKenna et al. [1] as a sensitizing device.

To achieve an understanding of CBs perception and practice of ICT, a mobile ethnographic study was adopted. Because the left side of the McKenna et al. model was based on propositions, they do not imply causality or directionality (as a quantitative model might). Therefore, we decided to perform an exploratory analysis which is recommended alongside a quantitative study in mixed method research [17, $18]$.

Three ethnographic studies were undertaken between June 2014 to December 2014 by following three different groups of $\mathrm{CBs}$ in Europe using participant observations and in-depth interviews. Data was also collected through China's biggest online travel forum Qyer.com, instant messenger, social networking applications such as WeChat, as well as everyday practice of ICTs.

\section{Table 1: Study Informants' Demographics}

\begin{tabular}{|l|l|l|l|l|}
\hline Trip & Name & Sex & Age & Occupation \\
\hline \multirow{3}{*}{$\begin{array}{l}\text { Spain } \\
\text { Portugal }\end{array}$} & A-Wei & $\mathrm{M}$ & 28 & Architect \\
\cline { 2 - 5 } & Will & $\mathrm{M}$ & 24 & Graduate student \\
\cline { 2 - 5 } & Yixiu & $\mathrm{F}$ & 24 & Fashion \\
\cline { 2 - 5 } & Winly & $\mathrm{F}$ & 24 & Graduate student \\
\cline { 2 - 5 } & Zhang & $\mathrm{F}$ & 30 & Accountant \\
\cline { 2 - 5 } & Xu & $\mathrm{F}$ & 33 & Teacher \\
\cline { 2 - 5 } & Wang & $\mathrm{M}$ & 34 & Self-employed \\
\hline UK & Kitty & $\mathrm{F}$ & 40 & Marketing in Bank \\
\cline { 2 - 5 } & Aileen & $\mathrm{F}$ & $40+$ & HR in Bank \\
\cline { 2 - 5 } & Catherine & $\mathrm{F}$ & $40+$ & Cloth trading \\
\hline Poland & Jennifer & $\mathrm{F}$ & 30 & IT \\
\cline { 2 - 5 } & Jerry & $\mathrm{M}$ & 29 & IT \\
\cline { 2 - 5 } & Justin & $\mathrm{M}$ & $35+$ & IT \\
\cline { 2 - 5 } & Harry & $\mathrm{M}$ & 24 & Graduate Student \\
\hline
\end{tabular}

Informants (cf. Table 1) were approached and recruited through Qyer.com, which is currently the biggest Chinese outbound backpacker online forum. The researcher revealed his identity and asked for potential informants' consent before carrying on further studies. After recruiting informants from Qyer.com, the researcher travelled with three groups of CBs and kept track of their daily behaviour of technology usages and their perceptions.

The fieldwork consisted of three trips: The first trip was conducted in August and September 2014 in Spain and Portugal. The second trip was undertaken September to October 2014 in the UK. The third trip was undertaken in November 2014 in Poland. The mobile ethnography includes both netnography [19], multi-sited ethnography [20], and auto-ethnography [21] following CBs' intersecting pattern of movement between the physical and the virtual world. Virtual data included informants' posts on their social media, 
group chat histories and online travel journals were recorded as a source of data. Physical data included two in-depth interviews for each key informants who the researcher travelled with, field notes of conversational interviews and participant observation throughout three journeys, as well as interviews with other $\mathrm{CBs}$ the researcher met during the trip. Altogether twenty-six pages of interview transcripts and field notes were accumulated.

In addition, the researcher also immersed himself in these three journeys to provide alternative insights of interactions and observations of informants reflexively as both a researcher and a CB. In total, 20 in-depth interviews and detailed field notes, and informants' online activities were recorded and generated from these three fieldworks.

A two phased approach was used to test the left side of model of McKenna et al. [1]. Following from McKenna et al., a task based analysis (cf. Table 2 and the section 4.1.) was performed to determine how CB use different CIS types. This involved understanding the steps involved in using a CIS. The tasks were then able to be mapped to each of the four CIS types from the model to confirm the McKenna el al. propositions. This then further enabled us to explore the use and acceptance variables for each CIS type through a qualitative thematic analysis [22]. The analysis consisted of two of the researchers coding the data. Where codes differed between researchers these were discussed to come up with an agreed upon code.

\section{Findings}

Table 2: Assign Tasks to CIS Types

\begin{tabular}{|c|c|c|c|c|c|c|c|c|}
\hline \multirow[t]{2}{*}{ ID } & \multirow[t]{2}{*}{ Task } & \multicolumn{2}{|c|}{ Uncertainty } & \multicolumn{2}{|c|}{ Equivocality } & \multirow[t]{2}{*}{ CIS Type } & \multirow[t]{2}{*}{ Construct } & \multirow[t]{2}{*}{ Application } \\
\hline & & Low & High & Low & High & & & \\
\hline 1 & $\begin{array}{l}\text { Go to online travel } \\
\text { forum to look for } \\
\text { travel companion }\end{array}$ & & $\mathrm{X}$ & & $\mathrm{X}$ & Collaborative & $\begin{array}{l}\text { Social } \\
\text { Influences }\end{array}$ & Qyer.com \\
\hline 2 & $\begin{array}{l}\text { Use navigator to lead } \\
\text { direction }\end{array}$ & $\mathrm{X}$ & & $\mathrm{X}$ & & Computational & Self-Efficacy & $\begin{array}{l}\text { Google Maps } \\
\text { Navigator }\end{array}$ \\
\hline 3 & $\begin{array}{l}\text { Go to online travel } \\
\text { forum to look for } \\
\text { travel information }\end{array}$ & & $\mathrm{X}$ & $\mathrm{X}$ & & Networking & $\begin{array}{l}\text { Facilitating } \\
\text { Conditions }\end{array}$ & Qyer.com \\
\hline 4 & $\begin{array}{l}\text { Use word of mouth } \\
\text { applications to check } \\
\text { attractions, } \\
\text { restaurants nearby }\end{array}$ & $\mathrm{X}$ & & & $\mathrm{X}$ & Adaptive & Self-Efficacy & $\begin{array}{l}\text { TripAdvisor } \\
\text { Google Map } \\
\text { Rankings }\end{array}$ \\
\hline 5 & $\begin{array}{l}\text { Share travel } \\
\text { experience on social } \\
\text { media }\end{array}$ & & $\mathrm{X}$ & & $\mathrm{X}$ & Collaborative & $\begin{array}{l}\text { Social } \\
\text { Influences }\end{array}$ & WeChat \\
\hline 6 & $\begin{array}{l}\text { Reaching } \\
\text { companions }\end{array}$ & & $\mathrm{X}$ & $\mathrm{X}$ & & Networking & $\begin{array}{l}\text { Facilitating } \\
\text { Conditions }\end{array}$ & $\begin{array}{l}\text { Skype } \\
\text { Mobile Phone }\end{array}$ \\
\hline 7 & $\begin{array}{l}\text { Maintain } \\
\text { connectedness with } \\
\text { friends and families } \\
\text { when travelling }\end{array}$ & & $\mathrm{X}$ & & $\mathrm{X}$ & Collaborative & $\begin{array}{l}\text { Social } \\
\text { Influences }\end{array}$ & WeChat \\
\hline
\end{tabular}

To investigate our research model, we followed a similar approach of McKenna et al. [1] by analysing the tasks that CBs perform using technology to assign them to the CIS components and corresponding CIS types. Table 2 shows seven examples of the task level analysis. Following this, we will explore the use and acceptance variables for each task.

\subsection{Assign Tasks to CIS Types}

The first task involves CBs who cannot find travel companions in real life, go to online backpacker forums to look for travel companions. In this task, CBs either go to popular backpacker forums to make a post to assemble a travel group, or look for posts which have similar interests made by potential travel companions. It is suited to a collaborative service because the online forum allows users to produce information, and decision making of confirming travel companions, itineraries, budgets and bookings before the trip requires a process of negotiation. This task tends to have high level of uncertainty as Chinese backpackers do not have an idea who they will be travelling when looking for travel companions. In popular Chinese backpacker forums, there are many competing posts recruiting travel companions at the similar period of time. For backpackers who are looking for travel companions, there are many potential groups for them to join in; therefore, the equivocality of this task is high.

The second task involves applying mobile technologies such as a navigator or mobile application such as google map to lead the direction during 
backpacking. By doing so, CBs need to follow standardised and repeatable patterns to allocate current location, type in destination, choose the route, and follow the instruction given by the navigator. This task is suited to a computational service with low uncertainty as the navigator provides step-by-step instructions for backpackers to follow, and low equivocality as information of direction is simple and straight forward, so there is no chance of misinterpretation.

The third task involves $\mathrm{CB}$ go to online forum to acquire relevant travel information they need. They can either search by keyword on the website, or go into the section that their destination in to find posts which interest them. This task has high level of uncertainty as when backpacker entering the website, they do not know if they can find information they want or what type of information is available. On the other hand, this task has lower level of equivocality, as backpackers are less likely to have other interpretations of provided information. In this case, this task is suited to a networking service as the server produce and transfer one-way information by requests of backpackers.

The forth task involves CBs to use word of mouth applications on their mobile devices to look up restaurants, bars and attractions nearby. By following steps of these application provided, $\mathrm{CBs}$ are able to access to a list of restaurants or attractions that meet their criteria. CBs then make decision from the list based on the availability of the restaurant or attraction, and group preferences. It is suited to an adaptive service, as the number of conflicting restaurants or attractions, the diverse availabilities lead to the high equivocality. The uncertainty is low in this case, as these word of mouth application can provide comprehensive information of these restaurants.

The fifth task involves CBs sharing their travel experiences on their social media, in this case, mostly WeChat moment (similar to a social media post). These posts normally consist of combinations of pictures and text, some of them are mini-videos. The interactions of their friends and families as an essential part of this task, as the user is highly engaged in replying comments to extend their travel information sharing. This task is suited to collaborative service, as the $\mathrm{CB}$ co-produce information with their friends and families on social media through interacting under the post. The uncertainty is high as the user is out of control of the interaction flow, and the equivocality is also high in this task because the function of the WeChat moment, different user receives and interprets the information differently.

The sixth task involves CBs reaching their travel companions by using instant messenger or phone calls during the trip. This task is suited to a networking service as users produce information by confirming the location of each other by following certain pattern of technology usage. This task has high level of uncertainty as it can be only completed when facilities enable the connections and both parties participate; on the other hand, the task has low level of equivocality as the confirmation of location is straight forward and less likely to be incorrectly interpreted.

The seventh task involves CBs use WeChat instant messenger or WeChat Moment posts to maintain the updates and connectedness with their social groups back home. This task is suited to a collaborative service as the status of connectedness requires both parties to participate in producing information in an interactive manner. This task has high level of uncertainty because there are many uncertain variables that affect maintaining connectedness in the mobilities. In terms of the equivocality, the level is relatively high for this task, as the interaction flow and frequency do not follow repeatable pattern, and multiple social links can be active at the same time.

\subsection{Results of Qualitative Analysis}

This section will present our findings for the linkages of the service types to the acceptance and use variables. Through the task based analysis $(\$ 4.1)$, we found that all of the proposed links between CIS types and their usage variables were confirmed. From the qualitative analysis presented next we also found that there are new linkages not considered in the McKenna et al. model. Our results are presented in Figure 2.

\subsubsection{Collaborative CIS}

According to McKenna et al. [1] collaborative tasks are linked with social influences. We found this to be true. For example, in task 5, CBs share information online for their friends and family, and in particular they use WeChat to "check-in" to notify their parents of their safety during their travels. As one-child policy and collective culture have largely shaped Chinese society, it is crucial for CBs to update their safety, and mobile applications such as WeChat have made this a lot easier.

Jerry: "I will post some photos and check in the location, then all my friends on my social media know where I am." Or A-Wei: "I will do one post less than two days, let them know I am still alive. I tend to do it on WeChat moment than instant messenger."

But it is not only reporting of safety that has some social influences on collaborative services. For some, friends' 'like's and comments on online posts motivate CBs to share more. In addition, the feeling of 'being 
left behind others' was also a motivating factor for one informant to learn new features of WeChat, such as sending a 'sight' (a 6 second video).

Yixiu: "My friends' likes and comments motivate me to post more of my travel experiences." Or Cath: "I realised those core friends will interact with you frequently, even they do not comment, they will give me a like, actually I don't really care how many likes I receive, I care more about how they comment on my posts, that is real interactions, 'like' for me is just mean they have read it."

Field note: Cath started to research how to use the new function of 'sight', then she started to practice in our group chat. She said she cannot be left behind. She like to try new things.

Furthermore, we also found proof that the boundaries between service types are blurred as suggested by McKenna et al. [1]. For example, we found instances in the data where collaborative services (from task 5) were becoming networking services, and vice versa. For example: Jennifer: "It is just like a dairy for me, I don't care if other people read it or not, it is for my record." In task 5 we also found examples where the task began as a networking task, and then continued as a collaborative task. Or Aileen: "From my own perspective, there is a lot to learn from during the trip. Sometimes people have short memories. For me, this is my main reason to use WeChat moment - to help me note down things, some key moments and big issues. I did not expect there is so many feedbacks, or so many people are following me."

Additionally, we found that collaborative tasks which were also linked with the usage variables of facilitating conditions and self-efficacy. For facilitating conditions, when $\mathrm{CBs}$ go online to find travel companions (task 1), technical infrastructure such as an internet connection, and organizational infrastructure such as pre-existing discussion forums is required to enable users to connect with other users.

Jennifer: "In the evening I just went to an Internet café, made a post on Ctrip to look for travel companions."

Also in task 5 there were also technical requirements or limitations when collaborative services are used, such as needing GPS, Wi-Fi to connect to services such as checking-in a location or sharing information. WeChat also has a limitation in the number of photos which can be uploaded in a post, which means that CBs must carefully select the photos they wish to share in a post.

Field Note: Jennifer told me places she has been to, when we went to the restaurant, she connected to Wi-Fi to show me her posts on WeChat.
Jerry: "I will post some photos and check in the location, then all my friends on my social media know where I am" Or A-Wei: "I don't have reception, so I use WeChat to maintain contacts" Or Jennifer: "I will carefully select photos to post, nine photos for a post is enough"

There were differing opinions on the purchasing of a local mobile sim card during their travels. For some it was important for safety, but for others it created an environment of isolation as they had no connection to the outside word when outside a Wi-Fi zone.

A-Wei: "I feel 9/10 safe, without I will be 4/10. Without a sim card I will be worrying I cannot find the road, and have to do extra work, such as download offline map beforehand." Or Winly: "Personally I quite enjoy this moment of isolation...in everyday lives, it tends to be more stable and routine, so I will rely more on social media."

We also found that self-efficacy is linked to collaborative services.

Filed note: In terms of looking for travel companions, I went straight to Qyer.com, which is the most popular Chinese outbound backpacker forum. Hundreds of people looking for travel companions there every day. I believe I can find my travel companions and potential informants there.

\subsubsection{Computational CIS}

According to McKenna et al. [1] computational CIS are linked to self-efficacy. We found this to be true. Self-efficacy implies some confidence in using a service. In task $2, \mathrm{CBs}$ felt confidence in using navigational services such as an in-car navigator or Google Maps.

Cath: "I really like the navigator, I feel it is really handy, really boosts my confidence, in the future I don't need to worry about where I am going."

Field note: We got on the bus, although we did research last night, as this bus will not terminate at the salt mine, which means we have to get off at some point, it is a local bus and we don't understand Polish, we have to get the google map on to make sure we don't get off the wrong stop.

Our informants also illustrated confidence in using technology over traditional printed maps or asking for directions. One also suggested we are becoming more reliant on mapping services.

Field Note: A-Wei suggested: 'the local market suggested by the flat owner, shall we go and take a look?' we agreed, although we have the printed map, we still use Google Map to lead the way.

Cath: "Now I know how to use Google Map, I think I will rely on it a lot. Without it I can't not figure out how to get around. Before I know how to use Google 
Map, I will ask around, that is my first choice. I won't choose to look at the actual map, that is too difficult for me." (Question from interviewer: Do you think this kind of reliance is getting stronger and stronger?) "Yes, I believe so, I think it is a good thing, but it also scares me."

Additionally, we found that computational CIS are linked with social influences. We found that some members in the group were influential getting others to use certain services.

Field note: We followed google map to the station, with the mobile Wi-Fi with me, she seems to rely on me more than yesterday.

We also saw that computational CIS can be used across multiple applications.

Field notes: I followed the Google Map to see the cathedral, surprisingly the cathedral is still open. I went inside to walk around and use WeChat to tell AWei this information and send him my current location on WeChat. Or Kitty used Google Map to share the location on WeChat, I was amazed Google Map had this function, Kitty laughed at me.

Additionally, we found an example where both social influences and facilitating conditions being linked to computational services. One informant saw the researcher using Google Maps, and tried to install it herself, but could not activate it due to her lack of phone signal.

Field note: Aileen saw me use Google Map, also tried to download and sign up an account, however, they need to send a verification code to her mobile phone, she did not have signal, she had to give up.

It became clear that computational services are also linked with facilitating conditions. This became clear with problems that informants were having with the in-car navigator. Navigators rely not only on having a good GPS, but also that the mapping services on the navigator have been well designed (i.e. street names, directions). At times when the navigator would not work informants were forced to use other mapping services such as Google Maps. This can provide a set of different functions that may not be available on one service, but is available on another. At times informants also combined the use of both mapping services. Navigating services also may not always lead in the most direct route.

Field note: As navigator cannot recognize one of the road, I use Google Map instead to guide.

Li Yuming: "There was an accident one day, AWei's navigator had a problem. Then he becomes very anxious."

Field notes: For navigation, we mostly rely on the navigator kitty brought from China, but sometimes got supported by my google map. Or When we got on the car, the navigator seems not to corporate, it kept making mistakes, which must us take really a while to get out of the city. Or As these small villages do not have street names, they have to use Google Map instead to navigate. Or As Yixiu does not have local mobile sim card, and has never beeen alone before, so I was a bit worried she will get lost, I use my Google Map to plan the route for her, she took a photo with her phone, and asked for printed map, then she left.

\subsubsection{Adaptive CIS}

According to McKenna et al. [1] adaptive CIS are linked to self-efficacy. We found this to be true. Similarly, to the previous section, we argue that selfefficacy implies confidence. Informants also showed confidence in using adaptive services, in our example using word of mouth information services such as TripAdvisor, or a combination of applications to receive information.

Jerry: I use google map, and also TripAdvisor. Sometimes I use Wikipedia to check something. For instance, you need to do some background research for the places you are visiting. For example, some cultural sites, churches, otherwise it is not worth the trip.

In some instances, informants were more trusting of the information that receive from word of mouth services than face-to-face recommendations, or information from books which don't come with rankings, reviews or alternatives as apps like TripAdvisor provide.

Field notes: We didn't take recommendations from the host, but looked up from TripAdvisor to find a nice restaurant. Or We ignore the information book and tend to look at TripAdvisor.

However, we also found that too much selfefficacy could produce a reliance on technology, and we suggest possibly a lack of common sense or individuality.

Jennifer: "I can't travel without this phone. I have to download a local map before I travel." Or Kitty: "To be honest, I don't really enjoy this kind of reliance. Why it is the authority? Why we have to live with others 'opinions? Or making decision base on that?"

We found that adaptive services were also linked with social influences, as informants often found that their usage of the services was socially influenced.

Field notes: She asked me to go online to check any high rating restaurant nearby, I used TripAdvisor to check nearby restaurant. Or She keep asking me to use TripAdvisor. Or Before Kitty asked, I already started looking for places to eat on TripAdvisor. As expected she came to ask me to look up where to eat later.

Kitty: "In terms of ICT, this time I tend to rely more on different types of social medias and mobile 
technologies. WeChat is a must, I also use Google Map, City Mapper, TripAdvisor a lot. This is an era that making decision based on others' opinions."

We also found that adaptive CIS were linked with facilitating conditions. Facilitating conditions implies that there is information readily available online for users to access the services they need. In our examples, we found that often this information may be misleading, and users may act on this information only to be disappointed. For example, pictures uploaded may not be realistic, a similar sounding restaurant at a different location could turn out to be the same branch as another one, or reviews might not represent reality.

Field notes: Followed the TripAdvisor we arrived a restaurant, which has a similar name from yesterday but different rating on TripAdvisor. When we were seated and read the menu, we realized it actually is the same restaurant as yesterday we went, but a different branch. Or We found the restaurant, Yixiu said from the picture on TripAdvisor, it does not look nice. Or We finally found the café in Durness, according to TripAdvisor, the café has amazing hot chocolate and a great view, but the experience is actually quite different from what we expected.

\subsubsection{Networking CIS}

According to McKenna et al. [1] networking CIS are linked with facilitating conditions. We found this to be true. For example, in task 6, when travelling in an unfamiliar location, and a group gets separated, it is necessary to attempt to reach missing members. As roaming can be expensive, informants often buy local mobile SIM cards to be connected. However, there are often problems with this as mobile phone provides sometimes 'lock' phones so they will not accept a foreign SIM card, or they require PIN codes to unlock them.

Field notes: In the evening we cannot get hold of Will, but only he has the key of the flat, later he said he bought a sim card, but it got locked when he restarted the phone, and he left the PIN code in the apartment. Or as it is not that easy to find me, she has to reach me through her mobile number in China to do an international call.

Another example from task 3 is going online to look for travel information. If there is no information available, it can make a $\mathrm{CB}$ anxious as they tend to have a high level of risk avoidance.

Jerry: "Now I am still worried about how to get from the airport to the city center, in this kind of small place it is rather difficult to find official information, and from other people's experience sharing, there is no one certain conclusion."
These problems are also enhanced if there is no phone signal in a specific location. For example, task 6 confirms that self-efficacy is also linked with networking services. The facilitating conditions meant that users had no phone signal, and another group member used his ability (self-efficacy) to attempt to make contact through Skype (knowing that the others had a mobile Wi-Fi). This demonstrates that users are aware of conditions inhibiting information services, and will attempt other approaches to maintain connection.

Field note: I tried to call them, but the problem is the cottage we stayed in the Lake District has very bad reception. I tried to call them from my Skype, but the problem is I am not sure if they have reception on their phones or not. Tried to contact them so many times. Many calls connected for a few seconds then disconnected. I just know they got lost but don't know if they know the way back or not, and don't know how far away they are.

Self-efficacy is also demonstrated with users who know specifically what they are looking for (task 3 ), it can create a sense of purpose and confidence and a willingness to invest time.

Yixiu: "In terms of reading Qyer.com posts, normally I just focus on things I am interested, for instance the book store, I will spend extra time to look into it and find out the history and what it is for."

We also found that networking services are linked with social influences. For example, in task 3, informants go online to find information about where to travel, and base their routing decisions on what others have recommended. The use of online forums has strong social influences because in a group situation, some group members will encourage one person to make decisions. This requires research on routes and attractions. These kind of information services also encouraged one informant to travel more.

Winly: "I will go to Qyer.com to read other people's post, and some friends' recommendation, to see how design the route, then make decision to choose some popular destinations." Or Yixiu: "Sometimes I was reading the 10 most beautiful beaches in Portugal, but that was because we need to decide the route." Or Jennifer: "Yes yes yes, I printed them out, I feel this is so convenience, and so much fun! Since then I travelled much more."

\section{Discussion}

Our findings show that CBs mostly use collaborative CIS throughout their trips. Collaborative CIS are highly linked with social influences, which aligns with collectivistic and Confucius values of Chinese culture [7]. These CIS produce information 
by sharing travel information and updating safety status in backpackers' social circle.

The next most used CIS type was computational, which are highly linked to self-efficacy. This service type allows backpackers to fulfil the trip smoothly and relates to how $\mathrm{CBs}$ use technologies to assist themselves for travel related activities. This relates strongly to general backpacker culture which suggest independence and learning new skills throughout the trip. One key characteristic of backpackers is they are relatively information sensitive. With availabilities of the Internet, they are able to apply multiple tools and technologies to assist themselves to accomplish various tasks. For $\mathrm{CBs}$, they particularly prioritise 'learning through backpacking'.

Our findings also show that the least used services are adaptive and networking CIS. Adaptive CIS, which are also linked to self-efficacy, allow the CBs like to use technologies which enable them to integrate their daily lifestyle (from home) into their trip. These types of CIS need to be easy to use without the user needing to ask for help. Maintaining connectedness also enable $\mathrm{CBs}$ to get access to their mundane lives. Although most informants expected to escape their daily lives when backpacking, they faced challenges of this escapism when the Internet was connected. In networking services, CBs need to be able to reach each other through mobile technologies while travelling, which means facilitating conditions are very important. However, social influences are important as well as having the knowledge and capability to try alternative methods of connection if one fails.

Figure 2 summarises the findings and proposes a research model for further testing. The research model proposes to confirm the earlier results of McKenna et al. [1] in terms of providing further support for the links between adaptive, collaborative, computational, and networking CIS and a selected set of the UTAUT constructs: self-efficacy, social influences, and facilitating conditions. These links were confirmed by the combination of the task based analysis (section 4.1) and the qualitative analysis (section 4.2). These are represented as solid lines in Figure 2. Additionally, during the qualitative analysis, we discovered new links between CIS components and the UTAUT constructs, as represented by dashed lines in Figure 2. These links are:

- Adaptive CIS components are linked to Social Influences and facilitating Conditions.

- Computational CIS components are linked to Social Influences and facilitating Conditions.

- Collaborative CIS components is linked to SelfEfficiacy.

- Networking CIS components are linked to SelfEfficacy and Social Influences.

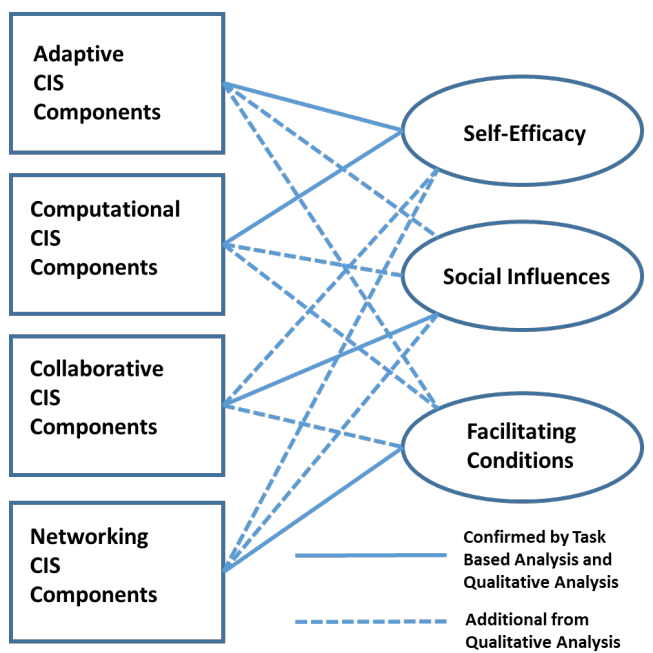

Figure 2: Findings and proposed research model.

\section{Conclusions}

This paper explores the link between CIS and technology usage to understand how users receive value from using ICT enabled services [3] by examining the relationship between CIS types and use and acceptance variables in an intercultural tourism setting applying mobile ethnographic research methods on Chinese outbound backpackers in four European countries. This study contributes primarily to the McKenna et al. model, and secondly to an understanding of CB IT services usage. It is the first paper to explore the relationship between the CIS and the use and acceptance variables by applying the model in an intercultural tourism context. The study also found that Chinese prefer to use mostly collaborative CIS which proves that Chinese collectivist values can be implemented through ICT.

Furthermore, we analysed against multiple CIS whereas the McKenna et al. [1] paper developed the model through the use of one mobile application. From a user's perspective, this paper shows a complex picture of blurring boundaries among three use and acceptance variables that user receive when using different CIS. This paper provides evidence of additional relations between CIS types with use and acceptance variables, which were not proposed by the McKenna et al. [1] model. This suggests that for designing different services types, self-efficacy, social influences and facilitating conditions should be all taken into account. This paper also explores the blurring boundaries among four CIS types and that from the perspective of a user, it is more likely to integrate multiple CIS types in one single task.

We also recognize that our study has some limitations. First of all, we only studied the CIS from the perspective of Chinese culture. We also 
acknowledge that Chinese backpackers have very strong characteristics and cannot fully represent tourists in general (i.e. non backpacker tourists). The applied theoretical model has also some limitations. The McKenna et al. [1] model does not consider other UTAUT variables such as anxiety. We do have some data about users panicking/anxious when CIS might not work. However, for this study we did not include this in the current study as the anxiety construct was removed from the model by [1]. Consequently, the use context of CIS should be further taken into consideration with future research. This concurs with Tuunanen et al. [4] argument that the use context impacts CIS use.

Future research should further develop the proposed research model through a quantitative analysis. The additional links discovered through the qualitative analysis also need to be further tested. The model can be used to compare with other cultures as others might have different CIS usage behaviour/patterns, but also to look at usage and/or design of CIS for specific contexts based on the CIS types and their usage variables as presented in this study, i.e. tourism in general, business related contexts. This will enable us to explore the blurring of boundaries between collaborative and networking CIS further.

\section{References}

[1] B. McKenna, T. Tuunanen, and L. Gardner, "Consumers' adoption of information services," Information \& Management, vol. 50, pp. 248-257, 2013.

[2] M. Castells, The Internet galaxy: Reflections on the Internet, business, and society: Oxford University Press, 2002.

[3] M. Barrett, E. Davidson, J. Prabhu, and S. L. Vargo, "Service innovation in the digital age: key contributions and future directions," MIS Quarterly, vol. 39, pp. 135-154, 2015.

[4] T. Tuunanen, M. Myers, and H. Cassab, "A conceptual framework for consumer information systems development," Pacific Asia Journal of the Association for Information Systems, vol. 2, p. 5, 2010.

[5] G. M. Chen, "The impact of harmony on Chinese conflict management," in Chinese conflict management and resolution, G. M. Chen and R. Ma, Eds., ed Westport: Ablex Publishing, 2002, pp. 3-17.

[6] L.-H. Lin, "Cultural and Organizational Antecedents of Guanxi: The Chinese Cases," Journal of Business Ethics, vol. 99, pp. 441-451, 2011.

[7] Y. Reisinger and L. Turner, "Cultural Differences between Mandarin-Speaking Tourists and Australian Hosts and Their Impact on Cross-
Cultural Tourist-Host Interaction," Journal of Business Research, vol. 42, pp. 175-187, 1998.

[8] F. L. K. Hsu, "Psychosocial Homeostasis and Jen: Conceptual Tools for Advancing Psychological Anthropology," American Anthropologist, vol. 73, pp. 23-44, 1971.

[9] F. K. G. Lim, "Donkey Friends' in China: The Internet, Civil Society, and the Emergence of the Chinese Backpacking Community," in Asia on Tour, T. Winter, P. Teo, and T. C. Chang, Eds., ed: https://works.bepress.com/franciskhekgee lim/12 /, 2009.

[10] J. G. Molz, "'Watch us Wander': Mobile Surveillance and the Surveillance of Mobility," Environment and Planning A, vol. 38, pp. 377393, February 1, 20062006.

[11] N. Green, "Who's Watching Whom? Monitoring and Accountability in Mobile Relations," in Wireless World: Social and Interactional Aspects of the Mobile Age, B. Brown, N. Green, and R. Harper, Eds., ed London: Springer London, 2002, pp. 32-45.

[12] G. Cooper, "The Mutable Mobile: Social Theory in the Wireless World," in Wireless World: Social and Interactional Aspects of the Mobile Age, B. Brown, N. Green, and R. Harper, Eds., ed London: Springer London, 2002, pp. 19-31.

[13] L. Mathiassen and C. Sørensen, "Towards a Theory of Organizational Information Services," Journal of Information Technology, vol. 23, pp. 313-329, 2008.

[14] V. Venkatesh, M. G. Morris, G. B. Davis, and F. D. Davis, "User Acceptance of Information Technology: Toward a Unified View," MIS Quarterly, vol. 27, pp. 425-478, 2003.

[15] G. Walsham, Interpreting Information Systems in Organizations. Chichester: Wiley, 1993.

[16] H. Klein and M. Myers, "A Set of Principles for Conducting and Evaluating Interpretive Field Studies in Information Systems," MIS Quarterly, vol. 21, pp. 67-93, 1999.

[17] V. Venkatesh, S. A. Brown, and H. Bala, "Bridging the qualitative-quantitative divide: Guidelines for conducting mixed methods research in information systems," MIS quarterly, vol. 37, pp. 21-54, 2013.

[18] J. W. Creswell and V. L. P. Clark, Designing and conducting mixed methods research. London: Wiley, 2007.

[19] R. V. Kozinets, Netnography: Doing ethnographic research online: Sage Publications, 2010.

[20] G. E. Marcus, "Ethnography in/of the world system: The emergence of multi-sited ethnography," Annual review of anthropology, pp. 95-117, 1995.

[21] L. Anderson and M. Austin, "Auto-ethnography in leisure studies," Leisure Studies, vol. 31, pp. 131146, 2012.

[22] J. Saldaña, The coding manual for qualitative researchers. London: Sage, 2009. 